\title{
Aplicación del portafolio grupal en la asignatura de Dirección de Producción y Logística: resultados de una prueba piloto
}

\author{
Application of the group portfolio in Production Management and \\ Logistics subject: results of a pilot project
}

Sofía Estelles-Miguel (soesmi@omp.upv.es)

Teresa Barbera Ribera (mabarri@upvnet.upv.es)

Carlos Manuel Dema Pérez (cmdema@omp.upv.es)

Universitat Politècnica de València (España)

http://dx.doi.org/10.12795/EDUCADE.2013.i04.09

\begin{abstract}
RESUMEN: Aprovechando el proceso de adaptación al nuevo Espacio Europeo de Educación Superior (EEES) de la Universidad Politécnica de Valencia (UPV), los autores del presente artículo decidieron realizar una experiencia piloto en asignaturas de la licenciatura. Dicha experiencia, consistía en la elaboración de un portafolio electrónico por grupos de alumnos. El resultado esperado es que los alumnos llevasen más al día la asignatura y se ejercitasen con problemas similares a los del examen. Se quería comprobar si el cambio metodológico repercutía en el progreso de los alumnos. El presente artículo presenta la experiencia de innovación docente implantada en la asignatura de Dirección de Producción y Logística en el curso académico 2011-2012 de cuarto curso de la Licenciatura de Administración de Empresas. Los resultados obtenidos servirán para el diseño de una asignatura de grado similar de segundo curso en el mismo centro.
\end{abstract}

PALABRAS CLAVE: Espacio Europeo de Educación Superior, Innovación Educativa, Programa piloto, Metodologías activas, Portafolio electrónico.

\begin{abstract}
Taking advantage of the process of adaptation to the new European Higher Education Area (EHEA) of the Universidad Politécnica de Valencia (UPV), the present paper authors decided to conduct pilot projects in Bachelor subjects. This experience consisted in the development of an electronic portfolio by groups of students. The expected outcome is that students brought up the subject and they will practice with problems similar to the test. We wanted to see if methodological change impacted on student progress. This paper presents an experience of educational innovation introduced in the Management Production and Logistics subject, in 2011-2012 academic year, in the fourth year of the Bachelor of Business Administration. The results obtained will be used for the design of a similar Degree subject in the second course in the same center.
\end{abstract}

KEYWORDS: European Higher Education Space, Educational innovation, Pilot, Active methodologies, Electronic portfolio.

\section{INTRODUCCIÓN}

La adaptación al EEES implica diseñar las asignaturas en competencias y en objetivos de aprendizaje, esto afecta tanto a la metodología de enseñanza-aprendizaje como a la evaluación. Los docentes se ven obligados a replantearse la forma de impartir las clases, revisando si los métodos utilizados son los más adecuados para cada asignatura.

Artículo de investigación. Recibido: 30-05-13 - Versión revisada: 01-09-13, Aceptado: 17-09-13

Licencia Creative Commons BY NC ND $\cdot 2013 \cdot$ Asociación Española de Contabilidad y Administración de Empresas - AECA 
Otro cambio importante introducido por la EEES, ha sido centrar la atención no sólo en el aprendizaje teórico práctico de las asignaturas, sino también en el desarrollo de habilidades que preparan a los estudiantes para su integración en el mundo laboral. La gestión de las competencias plantea numerosas dificultades (Levy-Leboyer, 1997), y su consecución es lo que debe estimular a los docentes, para la aplicación de nuevas metodologías docentes con ayuda de las TIC (Clares, 2005) y (Garai et al., 2003).

Para ello, se necesita cambiar la dinámica de las clases tradicionales basadas en el aprendizaje memorístico de conocimientos, en otra donde los estudiantes sean una parte activa, pasando de ser sujetos pasivos a ser protagonistas y responsables de su propio aprendizaje (Zabala y Arnau, 2007).

Las asignaturas relacionadas con el ámbito de la empresa, pueden ser explicadas siguiendo metodologías muy diversas. Una de ellas, la forma clásica, ha venido generalmente marcada por una gran masificación de alumnos, exposiciones magistrales por parte del profesor y una evaluación basada en un examen escrito. En el caso de la presente asignatura, al haberse generalizado el uso de recursos tecnológicos se han podido utilizar otros tipos de metodologías docentes, aunque todavía no ha descendido el número de alumnos matriculados.

En esta línea, y a fin de que la transición al EEES fuera gradual, la Facultad de Administración de Empresas (FADE) de la UPV estableció para algunas asignaturas de primer curso, un programa piloto de créditos ETCS (European Credit Transfer System) durante el curso 2009-2010. Se trata de un paso previo a la implantación obligatoria de las titulaciones de Grado para el curso 2010-2011, de acuerdo a la nueva legislación sobre estudios universitarios (Martínez y Marí, 2011). Los objetivos principales de este programa piloto son dos. Por un lado, aplicar nuevas metodologías activas de enseñanza-aprendizaje para, posteriormente, analizar su viabilidad en las nuevas titulaciones de Grado. Y, por otro lado, se pretende que el alumno adquiera un papel más activo en su propio aprendizaje, pasando a ser actor principal del proceso de enseñanza-aprendizaje. El programa piloto es, entre otras cosas, una iniciativa encaminada a mejorar los procesos educativos y el aprendizaje por parte del alumno, permitiendo valorar el coste de la implantación de los programas de innovación docente en aspectos tales como: recursos necesarios, cambios y esfuerzos a nivel institucional y del profesorado, y muy en particular, la respuesta al mismo de los alumnos y su efecto sobre el rendimiento académico.

De acuerdo con la filosofía de dicho programa piloto, pero utilizando alumnos de cursos superiores, los autores del presente artículo deciden realizar un proyecto piloto en una asignatura de cuarto curso de la Licenciatura (a extinguir), que se define más ampliamente en el punto 2 del presente artículo y analizar de qué forma puede influir este cambio de metodología y evaluación mediante la utilización del portafolio electrónico, tanto en el aprendizaje de los alumnos, como en el porcentaje de aprobados, así como en las notas de la misma.

El presente trabajo forma parte de un proyecto más amplio sobre la incorporación de nuevas metodologías docentes activas en el sistema de educación superior.

\subsection{OBjetivos}

En el presente trabajo se plantean los siguientes objetivos:

- Potenciar la autonomía del alumnado, el pensamiento reflexivo y crítico, el trabajo en equipo, las destrezas profesionales y la capacidad de autoevaluación.

- Adquirir y desarrollar de la capacidad de identificar, formular y resolver problemas relativos a los contenidos prácticos de la asignatura. 
- Realizar una evaluación continua, de forma que las cuestiones y problemas planteados por los alumnos se fueran corrigiendo conforme se iban realizando, para que los alumnos aprendiesen de sus propios errores.

- Lograr que los alumnos llevasen la asignatura al día.

- Lograr que las notas de los estudiantes mejorasen.

\section{EL PORTAFOLIO COMO METODOLOGÍA E INSTRUMENTO DE EVALUACIÓN}

La implantación de metodologías activas en el aula permite la formación en competencias del alumnado (González y Wagenaar, 2003), lo que exige la utilización tanto de nuevas formas de enseñanza-aprendizaje como de nuevos sistemas de evaluación, que se adapten a las nuevas directrices europeas. En este sentido, la utilización del portafolio como metodología de enseñanza-aprendizaje y evaluación nace como metodología alternativa (Colás et al., 2005; Klenowski, 2005; Armengol et al., 2009; García y Baena, 2009). El portafolio es considerado como la herramienta de gestión integral más importante de la que disponemos en los contextos educativos en la actualidad (Mateo, 2007).

No existe un modelo único de portafolio, al existir distintas modalidades en función de los objetivos que se persiguen con su desarrollo: de aprendizaje (portafolio del estudiante), de enseñanza (portafolio del docente) o profesional. En la innovación educativa que se ha realizado nos centraremos en el porfolio del estudiante, concretamente en el portafolio electrónico.

Los portafolios electrónicos han sido tratados por muchos autores en los últimos años (Kimball, 2002; Kösters, y Ritzen, 2003; Prendes y Sánchez, 2008; Gallego et al., 2009; Guasch et al., 2009 y Cabero et al., 2012) quienes consideran que los alumnos podrán demostrar sus competencias en el utilización de los mismos.

La evaluación por carpetas se concibe como un tipo de evaluación alternativa (Mateo y Martínez, 2005), lejos de la tradicional, ya que se basa en la negociación y en el intercambio comunicativo entre el profesor y el estudiante durante el desarrollo del portafolio. El alumno tiene un papel fundamental como persona activa en el proceso evaluativo, ya que tiene que ser consciente de su progreso en el aprendizaje identificando qué aspectos domina y cuáles tiene que mejorar. Esto le va a permitir aprender a planificarse a partir de las orientaciones del profesor y le facilitará el autoaprendizaje a la vez que puede compartir los recursos con sus compañeros.

Un portafolio es un instrumento de evaluación cuyo objetivo es mostrar trabajos o evidencias de consecución de objetivos personales o profesionales que, ordenados y presentados de una determinada forma, cumplen la función de potenciar la reflexión sobre cada una de las prácticas realizadas (Barberà et al., 2006). También se define como el conjunto de trabajos efectuados por el estudiante durante su proceso de aprendizaje con los que puede identificar, expresar y evaluar sus dificultades, destrezas, habilidades y carencias, demostrando los aprendizajes adquiridos (García y Morillas, 2011).

El desarrollo de trabajos puede suponer un adecuado complemento a las clases teóricas y prácticas, al transmitir al alumno no sólo conocimientos sino también la posibilitad del desarrollo de habilidades y destrezas (Flórez y Albelda, 2012).

En un entorno virtual, las ayudas proporcionadas por el profesor juegan un papel importante a la hora de que el alumno o grupo de alumnos realicen su portafolio (Colomina y Rochera, 2002).

Este instrumento, presenta grandes ventajas, destacar que sirve como instrumento que facilita la reflexión del alumno o alumnos acerca de tu propia evolución en los conocimientos, por tanto, contribuye a la autoevaluación, ya que supone un proceso 
de reflexión de la propia práctica, en donde el sujeto realiza esa reflexión con el fin de mejorar su práctica y así seguir aprendiendo y formándose y por otro lado supone un cambio a la hora de concebir la enseñanza-aprendizaje (Cano, 2005). Para Monclús (2010) presenta un gran valor por su capacidad de estimular la reflexión y experimentación, suministrando información muy valiosa acerca del proceso de aprendizaje, así como del desarrollo de dicho proceso.

En nuestro caso, se ha utilizado el portafolio electrónico por la comodidad que supone tanto para el alumno como para el profesor, y la facilidad de acceso, ya que la UPV, dispone de una plataforma electrónica llamada POLIFORMAT donde existen carpetas electrónicas, en las cuales se puede ir recogiendo el portafolio por parte del alumno, y corrigiéndolo y devolviéndolo a la misma plataforma por parte del profesor.

\section{DISEÑO Y METODOLOGÍA}

Cabría indicar que dado que la docencia se desarrolla en una Universidad Politécnica, la utilización de las nuevas tecnologías está muy extendida, y la actitud, así como la formación de los profesores facilitan en gran medida su implantación (Paredes y Estebanell, 2005). Así, que prácticamente desde que se puso en marcha la intranet en la universidad el medio para poner a disposición de los alumnos los apuntes, materiales de apoyo, artículos científicos, problemas, etc... ha sido la web, por lo que si se compara, está con otras Facultades, nuestra situación es envidiable (Marín et al., 2011). En el caso de la UPV, en el curso 2002/03 se pone en marcha una plataforma educativa a través de la web, que facilita y estimula el empleo de nuevas estrategias de trabajo a través de foros, los chat, y también de nuevas formas de evaluación. La introducción de esta plataforma y su uso, resultado de las nuevas tecnologías ha constituido un punto de inflexión que nos ha llevado a plantear el presente estudio.

El carácter práctico de esta materia resulta adecuado para la implantación de la metodología docente activa de grupos de trabajo con competencias poliformativas.

\subsection{CONTEXTO Y MUESTRA}

La innovación educativa se realiza en la asignatura Dirección de Producción y Logística (DPL) que se imparte en el segundo semestre del cuarto curso de la Licenciatura de Administración de Empresas de la UPV, dicha asignatura es troncal y consta de 9 créditos, que se reparten de la siguiente forma 4,5 créditos de teoría de aula, 3 créditos de práctica de aula y 1,5 créditos de prácticas de laboratorio. En los últimos años se han tenido matriculados de media entre 200 y 300 alumnos por año. Esta asignatura va a ser transformada en la asignatura obligatoria Dirección de Producción y Operaciones (DPO) que se imparte en el primer trimestre del segundo curso de Grado en FADE de la UPV, que consta de 6 créditos ECTS que se reparten en 3 de teoría y 3 de práctica. El curso pasado, que fue el primer curso hubo 127 alumnos matriculados.

Tradicionalmente esta asignatura, ha generado un elevado porcentaje de suspensos y por lo tanto de alumnos repetidores, con el subsiguiente fracaso en la incorporación de las competencias propuestas. Esta situación ha llevado a los profesores de esta asignatura a replantearse en numerosas ocasiones el enfoque dado a la docencia de las mismas, con la finalidad de mejorar el proceso de enseñanza-aprendizaje y, por tanto, incrementar los resultados académicos de los alumnos.

\subsection{MetOdología}

En la primera clase de la asignatura, se les explica a los alumnos en qué consiste la misma, las metodologías a utilizar y como van a ser evaluados. También se les indica que existe un trabajo voluntario, a realizar en grupos, mediante un portafolio grupal, 
con el que pueden obtener hasta un máximo de dos puntos extras. Se le explica en qué consiste el trabajo, es decir, a partir del tema 2, tal y como se vayan terminando de explicar los temas en clase, tendrán que plantear y resolver por grupos un problema y una cuestión, de cada tema, y subirlos a la plataforma POLIFORMAT, en la zona ESPACIO COMPARTIDO. Además se les indica que en la misma pueden encontrar ejemplos. Desde dicha plataforma serán revisados y evaluados, por los profesores de la asignatura. Esto servirá, para darles nota del trabajo y para que vayan aprendiendo a plantear y resolver problemas en grupo, y vean si tienen alguna dificultad en los mismos, siempre pueden pedir tutorías al profesor, en caso de cualquier dificultad. Al ir realizando y entregando el trabajo, conforme se va desarrollando el curso, evita que se vayan dejando todo para el final. También ayuda a irse preparando para el examen final, que consiste en problemas y cuestiones similares a las que ellos plantean y resuelven en el portafolio.

Los problemas que se habían detectado en años anteriores, era que había un elevado número de suspensos y no presentados debido a que los estudiantes, se dejaban la asignatura para el final, y aunque la materia no es excesivamente complicada, sí que es bastante extenso el temario y además se necesita tiempo para practicar cada tipo de problema. Con la metodología planteada se pretendía solucionar estos aspectos.

\subsection{EvaluaCión DE LA ASIGNATURA}

Hasta el momento la asignatura se ha evaluado de la siguiente forma:

- $80 \%$ Examen:

- Cuestiones teórico-prácticas: $50 \%$ de la nota del examen.

- Problemas: $50 \%$ de la nota del examen.

- $20 \%$ Prácticas:

- Estas se realizaban en el laboratorio y se debía entregar una memoria de las mismas que incluyese la realización y el análisis de los resultados obtenidos.

- Trabajo voluntario: Se puntúa entre 0 y 2 puntos, la puntuación obtenida se sumará a la nota obtenida entre el examen y las prácticas, siempre que esta última sea superior a 4,5 puntos sobre 10. Esta puntuación se utilizaba para un trabajo voluntario anterior al portafolio y también se va a utilizar para el portafolio electrónico.

En nuestra innovación educativa se ha implantado una experiencia piloto en la asignatura de DPL, mediante la implantación de de un portafolio electrónico que se realiza de forma grupal, para adaptarlo, no sólo a mejorar la nota, sino a practicar lo que se le va a exigir en el examen. El programa piloto establece nuevas metodologías docentes basadas en la participación activa del alumno frente a la exclusividad de la clase magistral del profesor $y$, de igual modo, establece nuevos métodos de evaluación fundamentados en una valoración continuada del esfuerzo realizado por el estudiante y de las competencias y habilidades adquiridas al finalizar el proceso de aprendizaje de la asignatura.

\subsection{INSTRUMENTOS DE EVALUACIÓN}

Al finalizar el curso, se realizaron dos tipos de encuestas a los estudiantes, para que dijeran como creían que podía haber influido este trabajo en sus resultados finales este cuestionario se realizo con escala dicotómica, y es el que se presenta en la Tabla 1 y las competencias genéricas o transversales que ayudaba a desarrollar el portafolio grupal, así como las opiniones de los estudiantes sobre el uso del portafolio, este 
cuestionario utilizaba escala Liker de 1 a 5, este cuestionario se presenta en la Tabla 2, y también tenía preguntas abiertas, sobre la consideración que merecía para los estudiantes la aplicación del portafolio electrónico grupal en la asignatura DPL, que se pueden ver en la Tabla 3.

A continuación se recoge la encuesta que se pasó entre los alumnos, tanto los que habían realizado el trabajo voluntario como los que no:

\section{TABLA 1. ENCUesta SOBRE El tRabajo de DPL}

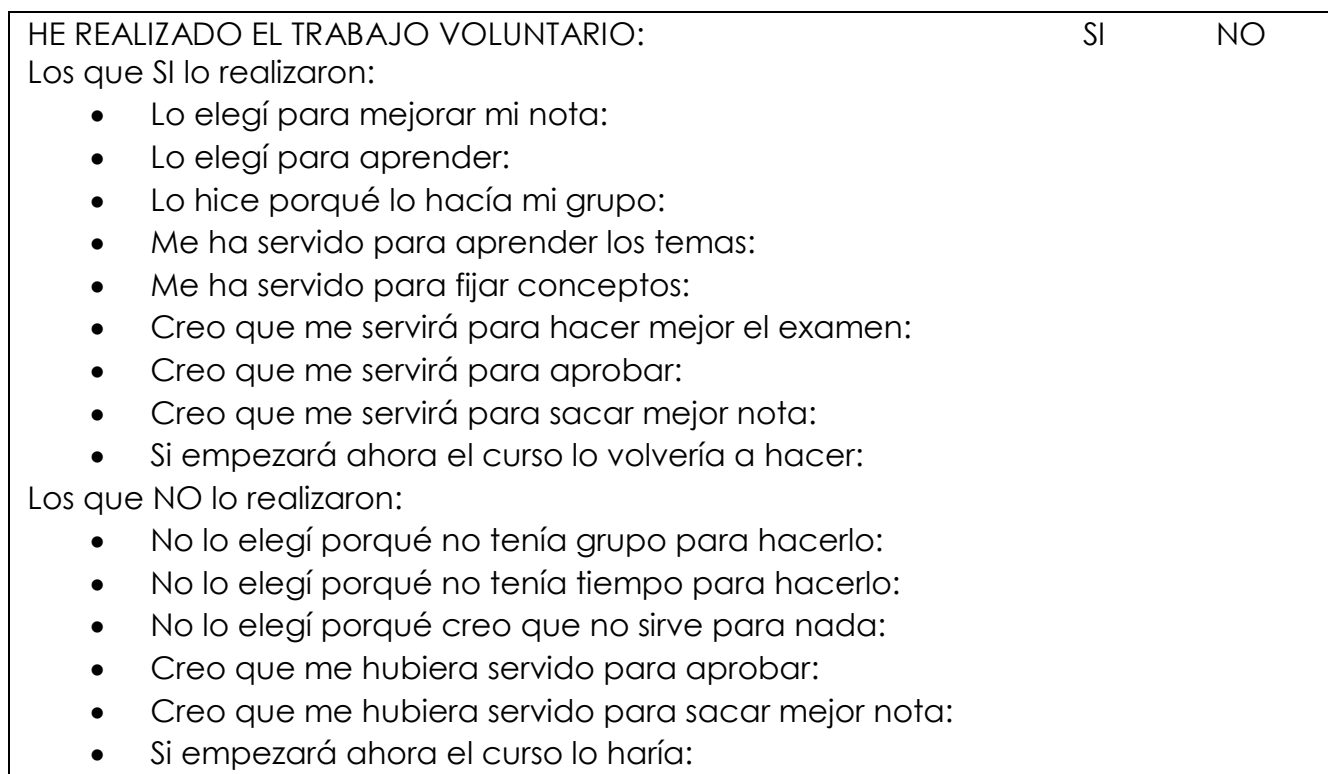

Fuente: Elaboración propia

TABLA 2. ESCALA LIKER SOBRE DESARROLLO DE COMPETENCIAS ADQUIRIDAS MEDIANTE LA UTILIZACIÓN DEL PORTAFOLIO ELECTRÓNICO GRUPAL

\begin{tabular}{|c|c|c|c|c|c|}
\hline COMPETENCIAS & 1 & 2 & 3 & 4 & 5 \\
\hline $\begin{array}{l}\text { 1. Fomenta la capacidad de organización y planificación } \\
\text { del trabajo }\end{array}$ & & & & & \\
\hline $\begin{array}{l}\text { 2. Facilita las relaciones interpersonales con los compañeros } \\
\text { y profesores }\end{array}$ & & & & & \\
\hline $\begin{array}{l}\text { 3. Potencia la autonomía, favoreciendo que el alumno } \\
\text { asuma un papel más activo en la construcción del } \\
\text { aprendizaje }\end{array}$ & & & & & \\
\hline 4. Desarrolla habilidades de trabajo en grupos interculturales & & & & & \\
\hline $\begin{array}{l}\text { 5. Ayuda a reflexionar sobre lo que se hace, como se hace, } \\
\text { dificultades encontradas y qué resultados se van logrando }\end{array}$ & & & & & \\
\hline 6. Favorece la motivación continua & & & & & \\
\hline 7. Capacidad de aplicar los conocimientos a la práctica & & & & & \\
\hline 8. Gestión del tiempo & & & & & \\
\hline
\end{tabular}

Fuente: Elaboración propia

La encuesta se realizó en la última clase, por lo que los alumnos todavía no habían realizado el examen y no podían saber si realmente esta actividad influiría en sus resultados. Así que los resultados obtenidos de la misma, se consideran únicamente en 
base a la apreciación de los alumnos. Lo contestaron los alumnos que acudieron a dicha clase, hubiesen realizado o no el portafolio electrónico.

Se utilizo otro cuestionario con una escala Liker de 1 a 5 , siendo la valoración utilizada la siguiente: $1=$ nada; $2=$ poco; $3=$ regular; $4=$ bastante y $5=$ mucho. Este cuestionario se acompañó al portafolio electrónico, por lo que lo contestaron todos los alumnos que habían realizado el mismo, junto con las preguntas abiertas que aparecen en la siguiente Tabla 3.

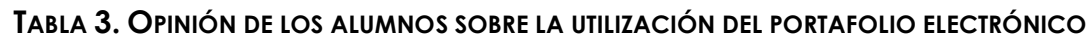

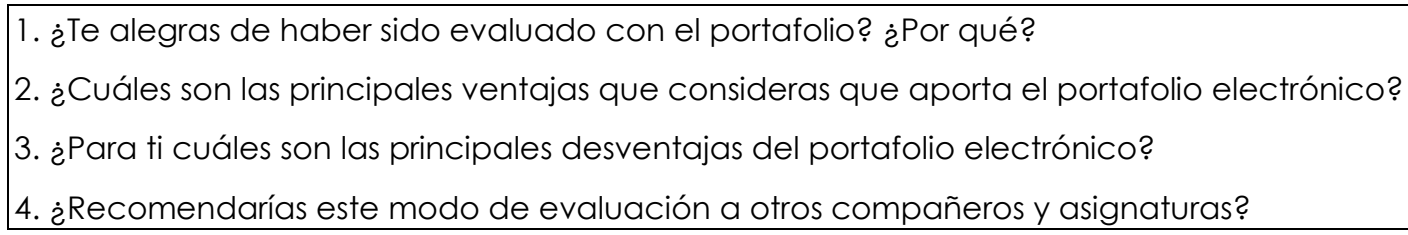

\section{RESULTADOS OBTENIDOS}

Aunque 74 alumnos habían realizado el portafolio electrónico, el cuestionario (Tabla 1) se pasó en la última clase de la asignatura, y al mismo contestaron 73 alumnos, que se reparten de la siguiente forma:

- 47 habían realizado el portafolio electrónico.

- 25 no lo habían realizado.

- 1 persona lo comenzó pero lo tuvo que dejar.

De los 47 alumnos que realizaron voluntariamente el porfolio electrónico grupal, se reflejan sus opiniones en los siguientes gráficos.

Gráfico 1. Lo ReALizARON PARA obtenER MEJOR NOtA.

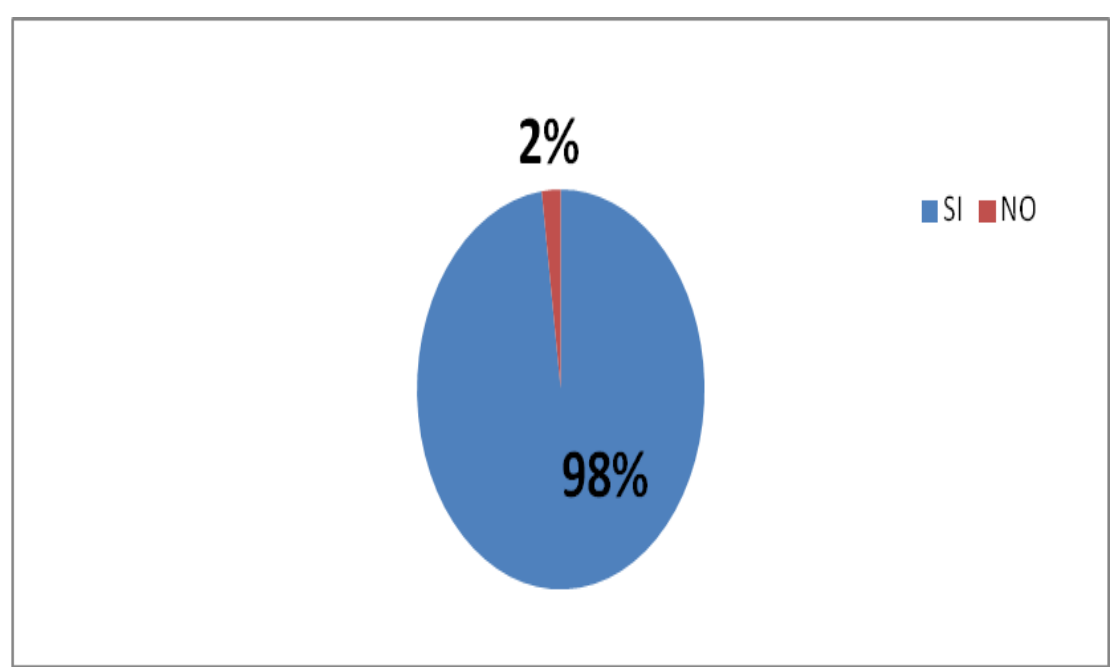

Fuente: Elaboración propia 
GRÁFICO 2. LO REALIZARON PARA APRENDER

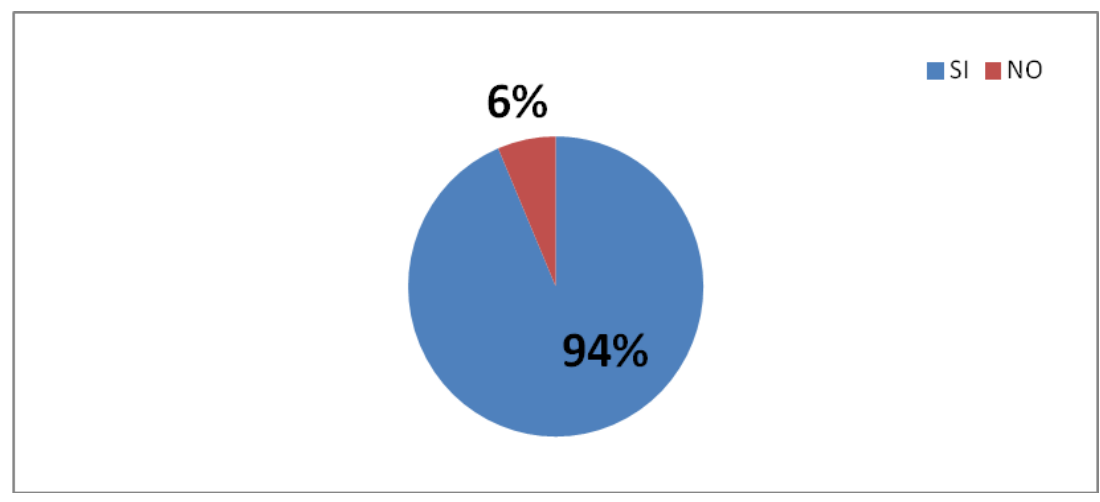

Fuente: Elaboración propia

GráFICO 3. LO REALIZARON PORQUE LO HACÍA SU GRUPO HABITUAL

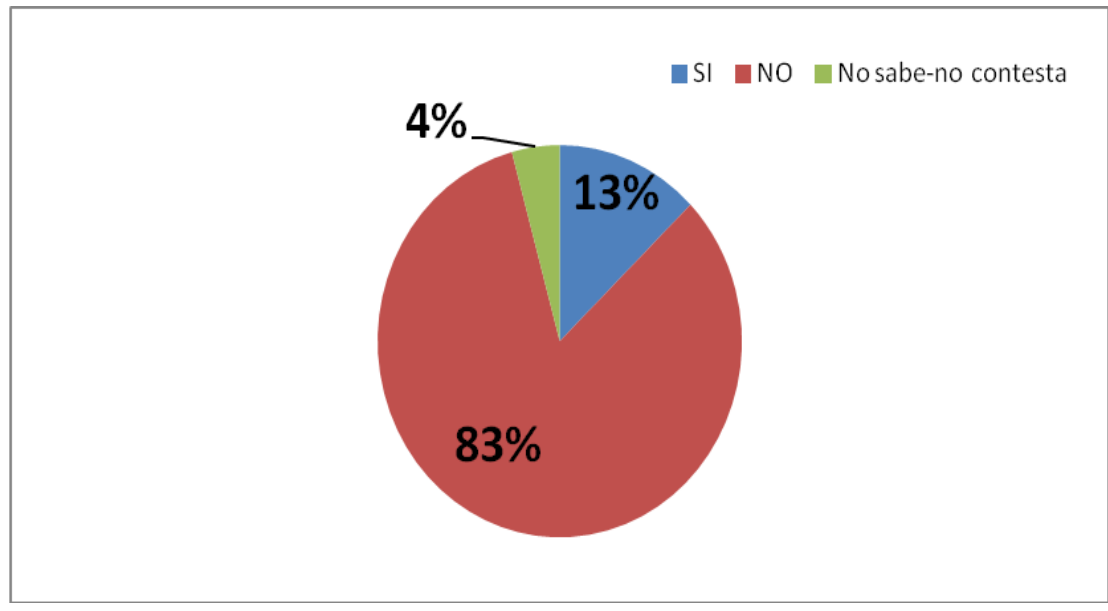

Fuente: Elaboración propia

GráfICO 4. PIENSAN QUE El PORTAFOLIO LES HA AYUDADO A APRENDER

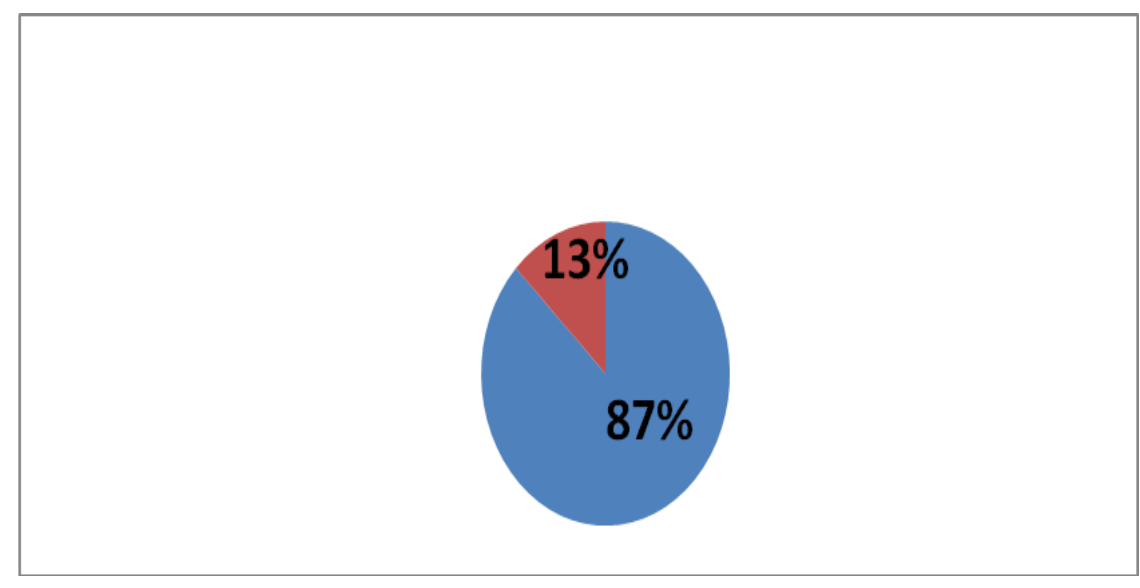

Fuente: Elaboración propia

educade, no 4, 2013, p. 131 
Sofía Estelles-Miguel, Teresa Barbera Ribera, Carlos M. Dema Pérez Aportación del portafolio grupal en Producción y Dirección logística

Gráfico 5. Piensan que el portafolio les ha AyUdado a fiJar conceptos

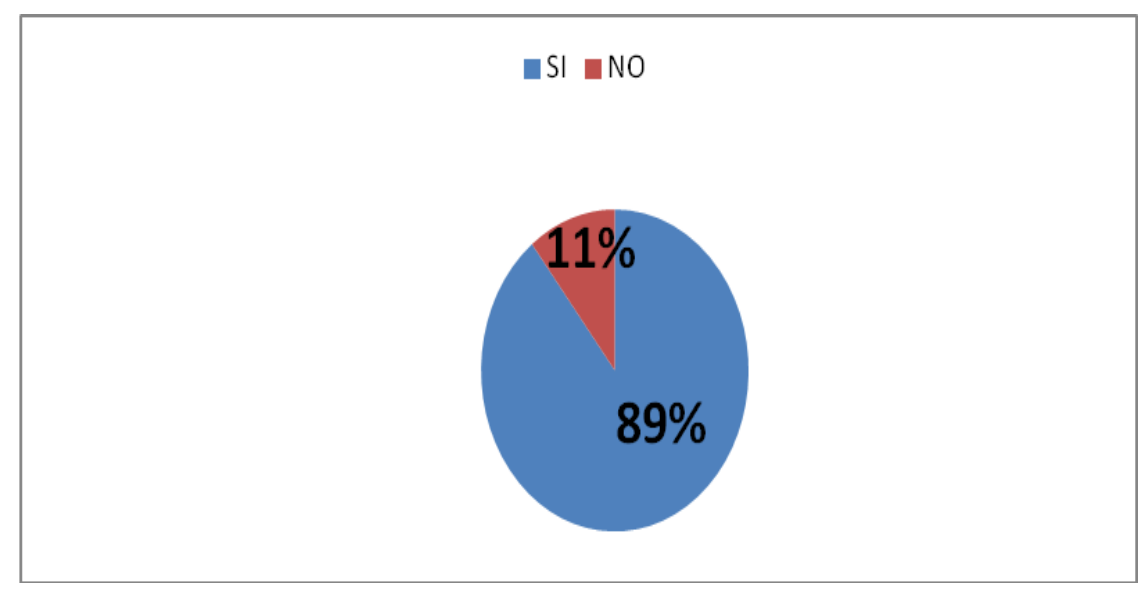

Fuente: Elaboración propia

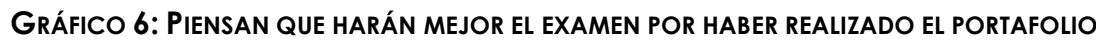

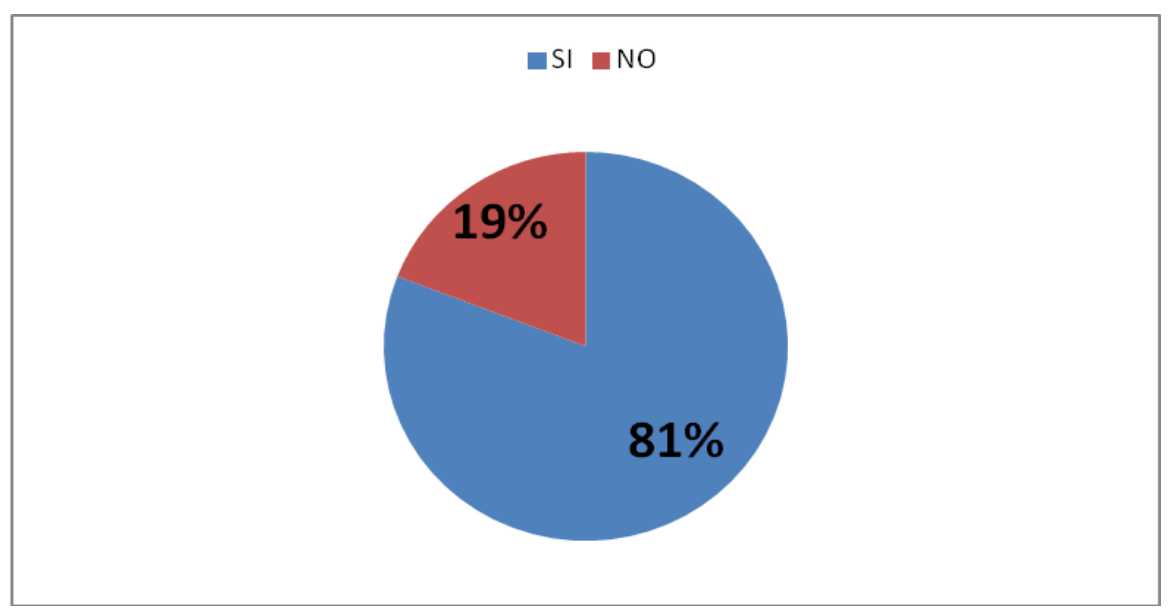

Fuente: Elaboración propia

Gráfico 7. PIensan QUe les Servirá para aprobar. Fuente: Elaboración Propia

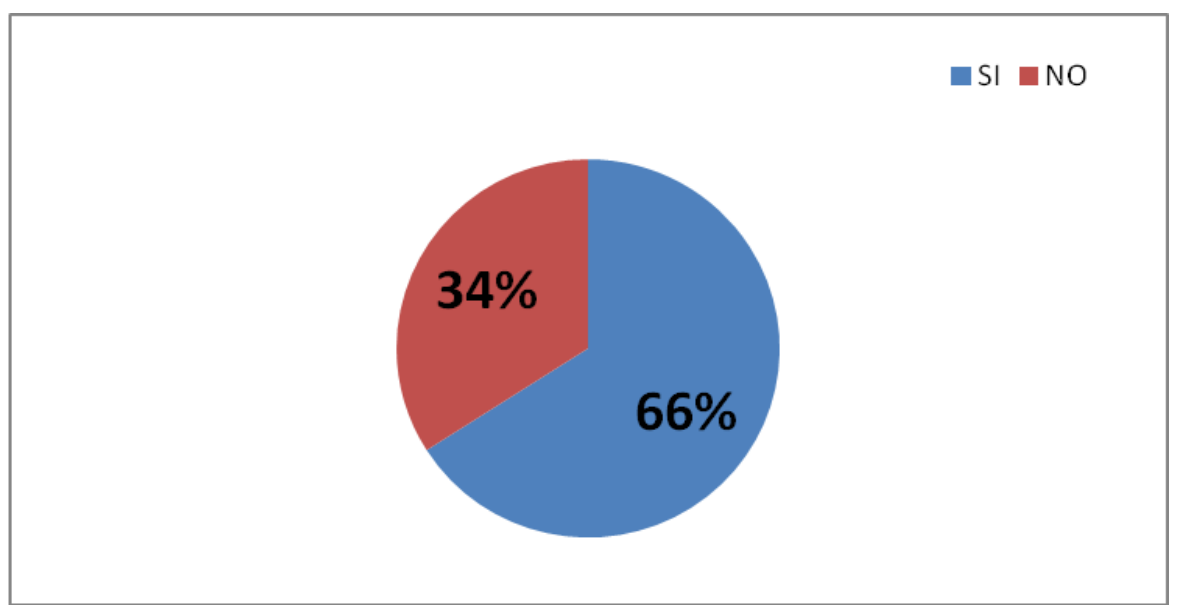

Fuente: Elaboración propia

educade, no 4, 2013, p. 132 
Gráfico 8. Piensan QUe les SerVirá para mejorar la nota

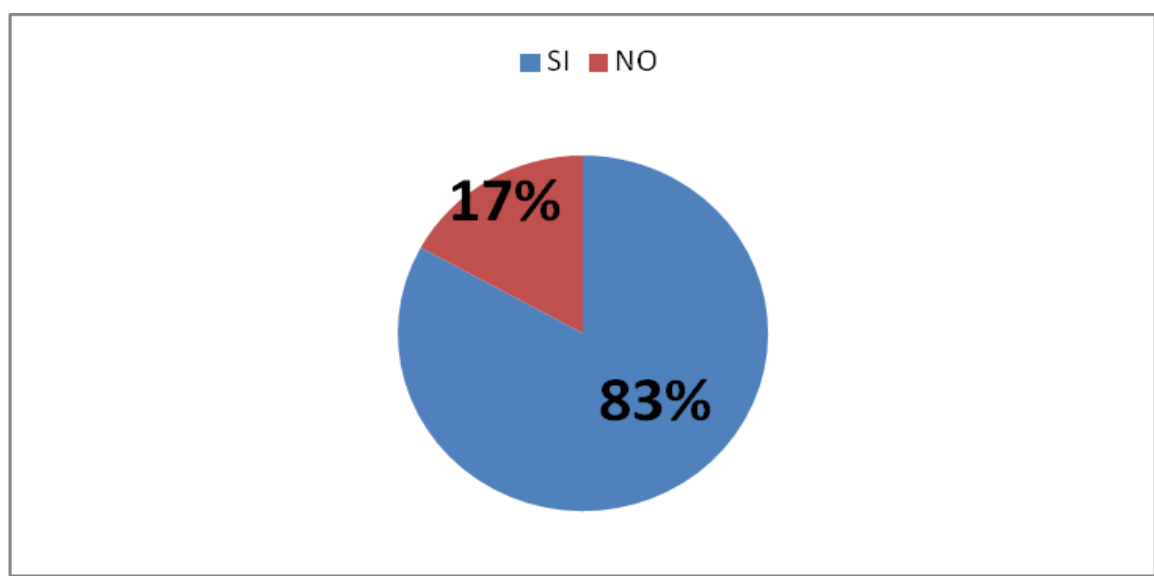

Fuente: Elaboración propia

GráFICO 9. Si EMPEZASE AHORA El CURSO HARÍA El PORTAFOLIO ELECTRÓNICO

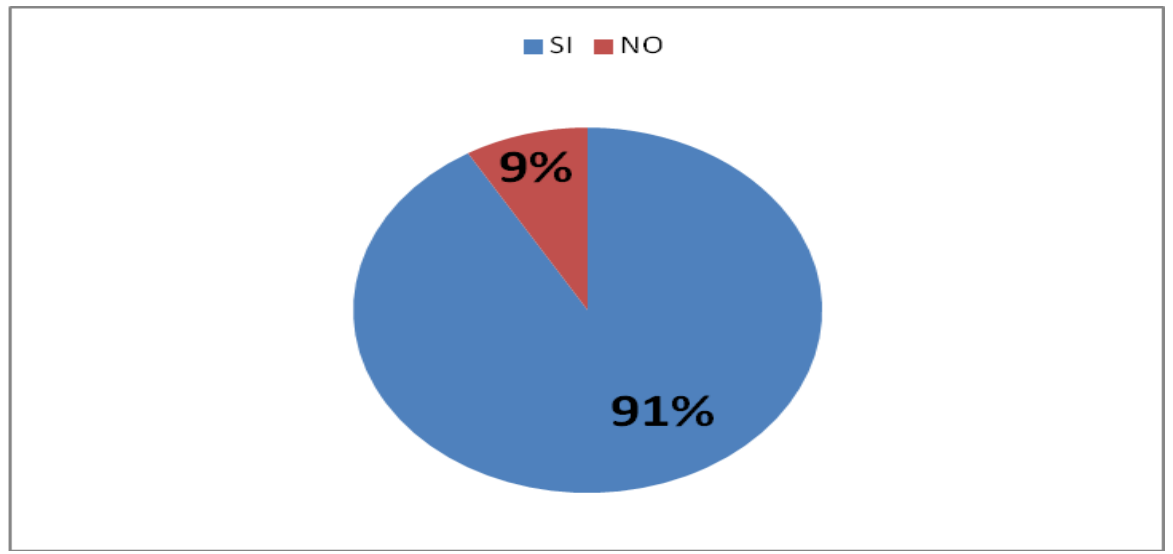

Fuente: Elaboración propia

Las contestaciones de los 25 alumnos que no realizaron el portafolio y que realizaron la encuesta de la Tabla 1, se recogen en los siguientes gráficos.

GRÁFICO 10. NO LO HICIERON PORQUE NO TENÍAN GRUPO CON QUIÉN HACERLO

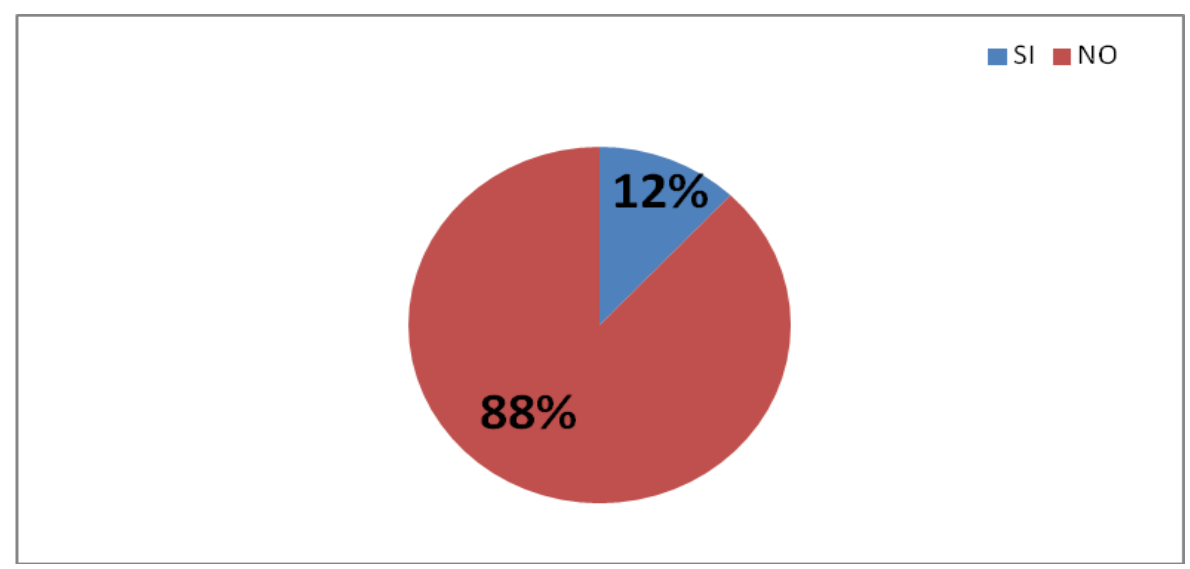

Fuente: Elaboración propia 
Sofía Estelles-Miguel, Teresa Barbera Ribera, Carlos M. Dema Pérez Aportación del portafolio grupal en Producción y Dirección logística

GRÁFICO 11. NO LO HICIERON PORQUE PENSABAN QUE NO SERVÍA PARA NADA

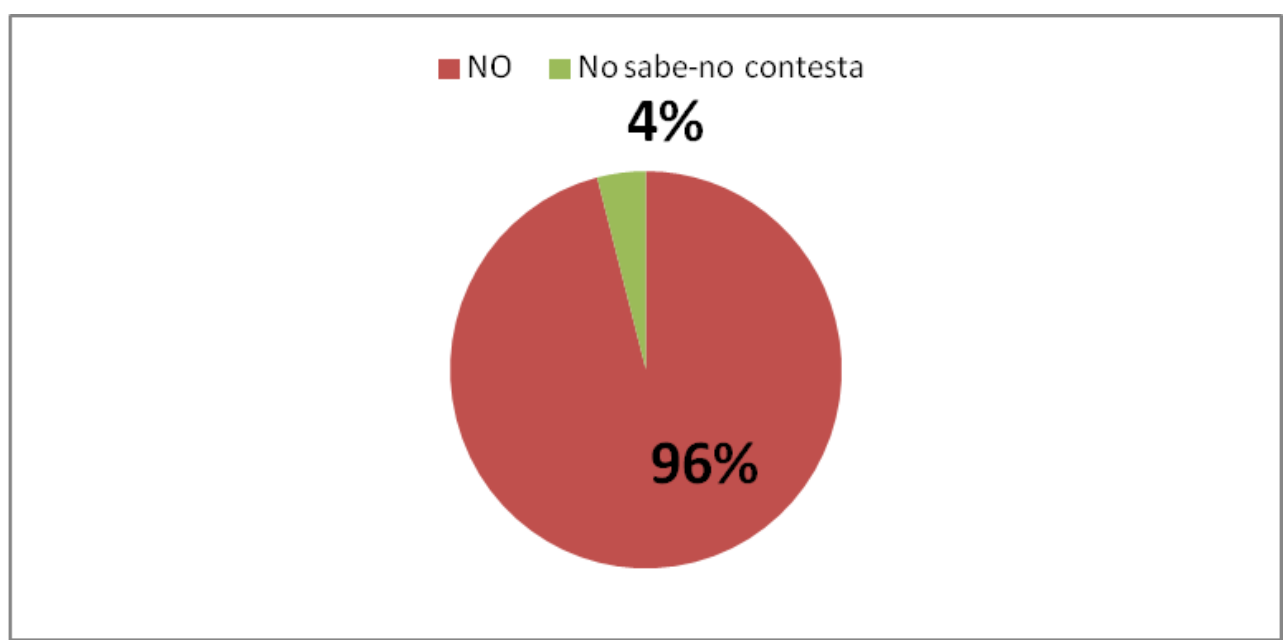

Fuente: Elaboración propia

Gráfico 12. Piensan que les hubiera servido para aprobar

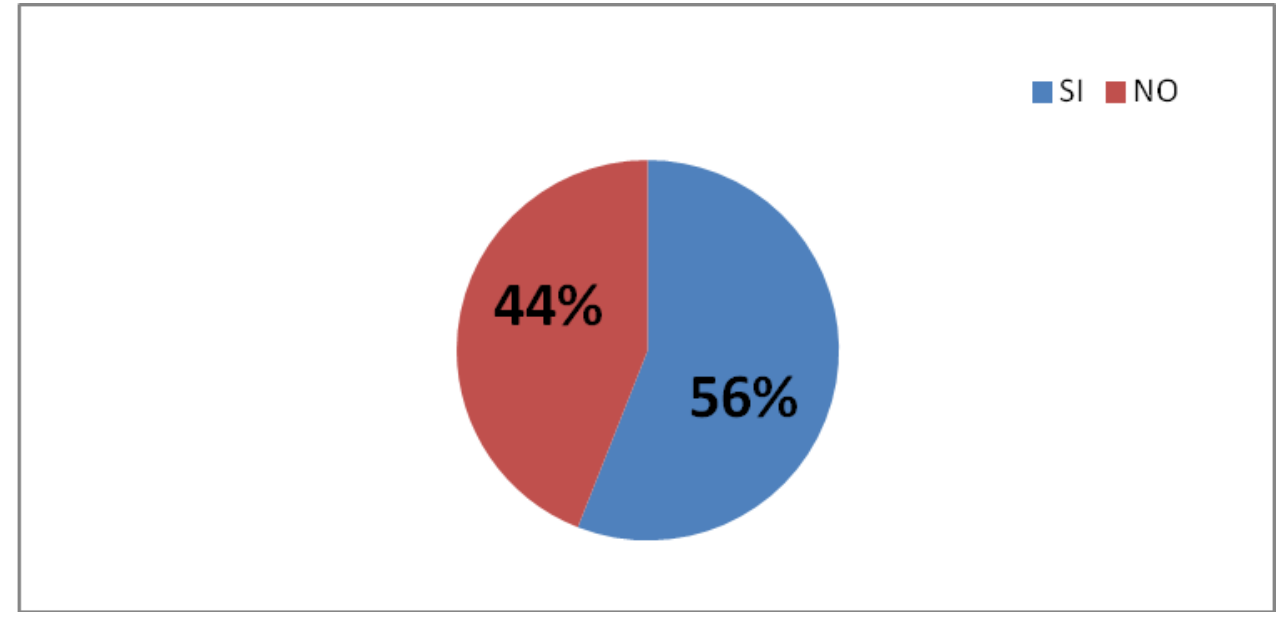

Fuente: Elaboración propia

Gráfico 13. Piensan que les hUbiera Servido para saCAR MEJOR NOta

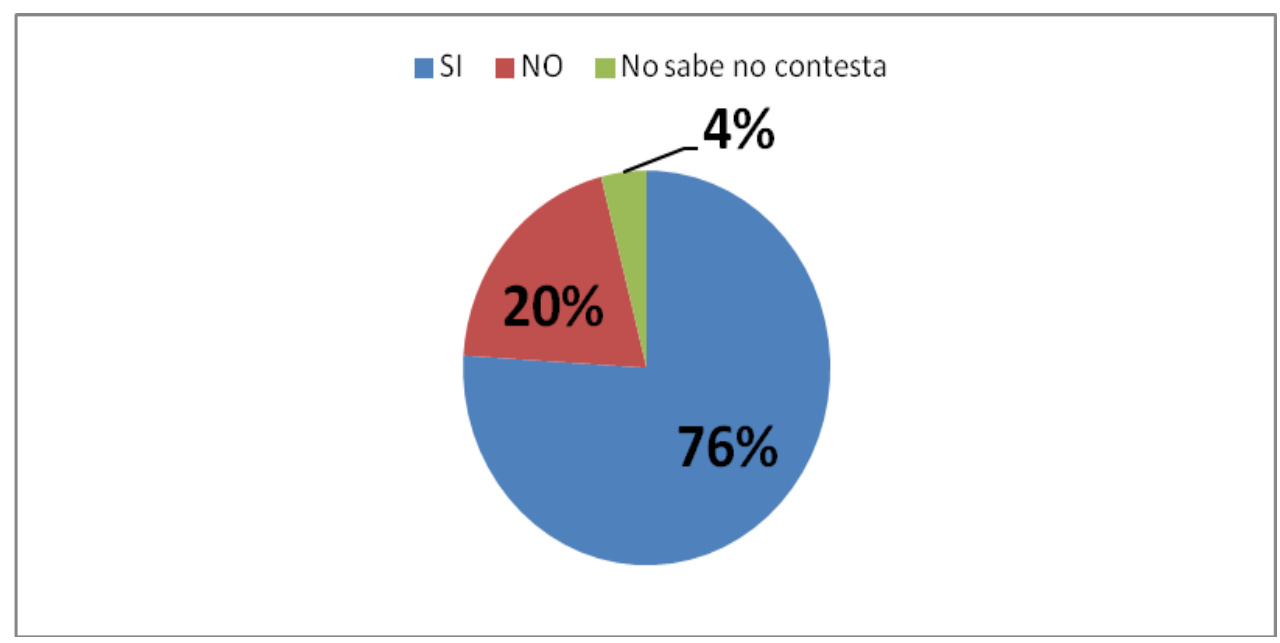

Fuente: Elaboración propia

educade, no 4, 2013, p. 134 
Gráfico 14. Si empezará ahora el CURSO lo haría

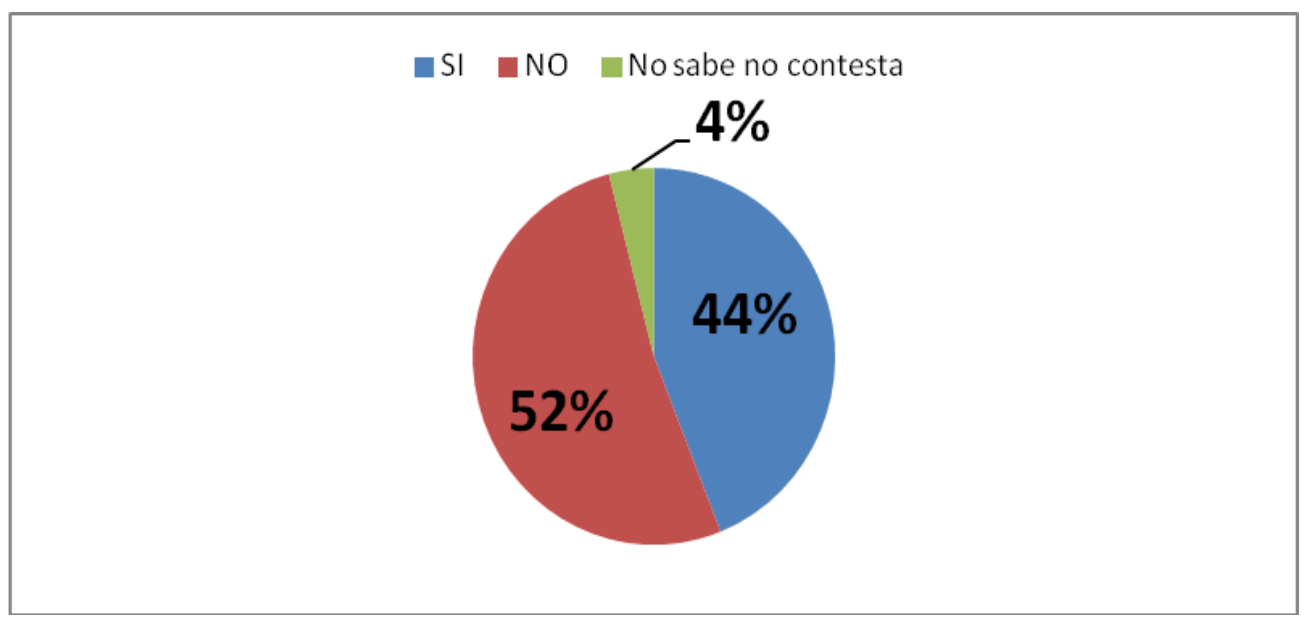

Fuente: Elaboración propia

En cuanto a la encuesta que se realizó junto con el portafolio electrónico (Tabla 2), se obtuvieron los siguientes resultados:

- Fomenta la capacidad de organización y planificación del trabajo: regular $(2,3 \%)$, bastante $(33,6 \%)$ y mucho $(74,1 \%)$.

- Facilita las relaciones interpersonales con los compañeros y profesores: regular $(19,3 \%)$, bastante $(39,1 \%)$ y mucho $(48,6 \%)$.

- Potencia la autonomía, favoreciendo que el alumno asuma un papel más activo en la construcción del aprendizaje: poco (4,6\%), regular $(26,3 \%)$, bastante $(35,4 \%)$ y mucho $(33,7 \%)$.

- Desarrolla habilidades de trabajo en grupos interculturales: regular (6,5\%), bastante $(40,2 \%)$ y mucho $(63,3 \%)$.

- Ayuda a reflexionar sobre lo que se hace, como se hace, dificultades encontradas y qué resultados se van logrando: regular (10,9\%), bastante $(38,3 \%)$ y mucho $(50,8 \%)$.

- Favorece la motivación continua: poco (7,5\%), regular $(26,4 \%)$, bastante $(47,9 \%)$ y mucho $(29,2 \%)$.

- Capacidad de aplicar los conocimientos a la práctica: bastante $(23,7 \%)$ y mucho $(76,3 \%)$.

- Gestión del tiempo: regular $(5,8 \%)$, bastante $(31,5 \%)$ y mucho $(62,7 \%)$.

Por tanto, las cuatro competencias más valoradas han sido: capacidad de aplicar los conocimientos a la práctica, capacidad de organización y planificación del trabajo, trabajo en grupos interculturales y gestión del tiempo.

Las principales contestaciones de los alumnos al cuestionario de preguntas abiertas (Tabla 3) han sido: gran cantidad de alumnos (65\%) se alegran de haber sido evaluados con el portafolio; las principales ventajas que aporta son ayudarles a estructurar su trabajo, ayudándoles a llevar la asignatura al día, el recibir feedback de los profesores, lo que facilita la preparación del examen; entre las principales desventajas se encuentran la cantidad de tiempo que requiere; y, por último, la mayoría de los estudiantes recomendarían su uso a otros compañeros y les gustaría utilizarlos en otras asignaturas. 
Sofía Estelles-Miguel, Teresa Barbera Ribera, Carlos M. Dema Pérez Aportación del portafolio grupal en Producción y Dirección logística

tabla 4. Comparativa de los datos obtenidos en DPL en dos Cursos consecutivos

\begin{tabular}{|c|c|}
\hline Curso 2010/2011 & Curso 2011/2012 \\
\hline Alumnos matriculados 190. & Alumnos matriculados 252. \\
\hline Alumnos presentados 150 (79\%). & Alumnos presentados 199 (79\%). \\
\hline $\begin{array}{l}\text { Alumnos que aprueban (junio+septiembre) } 88 \\
\text { (46\% sobre matriculados y } 59 \% \text { sobre } \\
\text { presentados). } \\
\text { - } 53 \text { alumnos en junio (60\%). } \\
\text { - } \quad 35 \text { alumnos en septiembre (40\%). }\end{array}$ & $\begin{array}{l}\text { Alumnos que aprueban (junio+septiembre) } \\
123 \text { ( } 49 \% \text { sobre matriculados y } 62 \% \text { sobre } \\
\text { presentados). } \\
\text { - } \quad 64 \text { alumnos en junio (52\%). } \\
\text { - } \quad 59 \text { alumnos en septiembre (48\%). }\end{array}$ \\
\hline $\begin{array}{l}\text { Distribución de las notas obtenidas: } \\
\text { - Sobresalientes: } 0 \text { alumnos (0\%). } \\
\text { - Notables: } 20 \text { alumnos (23\%). } \\
\text { - Aprobados: } 68 \text { alumnos (77\%). }\end{array}$ & $\begin{array}{l}\text { Distribución de las notas obtenidas: } \\
\begin{array}{l}\text { - } \\
\text { - }\end{array} \text { Nobresalientes: } 21 \text { alumnos (17\%). } \\
\text { - } \quad \text { Aprobados: } 62 \text { alumnos (34\%). }\end{array}$ \\
\hline
\end{tabular}

Fuente: Elaboración propia

\section{CONCLUSIONES}

En la experiencia realizada, se ha demostrado que, en la elaboración del portafolio electrónico grupal realizado de forma voluntaria, los alumnos administran y gestionan el trabajo, estableciendo pruebas significativas de su aprendizaje y de su competencia en habilidades claves.

La realización del trabajo, no ha mejorado el índice de presentados al examen. Este se mantiene constante en el $79 \%$ sobre alumnos matriculados en la asignatura. Se han mejorado los porcentajes de aprobados tanto sobre matriculados como sobre presentados en aproximadamente un $2 \%$, es una mejora, muy leve.

Lo que sí que parece que ha mejorado bastante, son las notas obtenidas por los alumnos, los sobresalientes ascienden en un 17\%, los notables en un $11 \%$ y baja el índice de aprobados rasos.

Con los resultados obtenidos se puede decir, que el cambio en el método de trabajo y el sistema de evaluación, si bien no ha servido para mejorar el índice de presentados y poco el número de alumnos que ha superado la asignatura, si que ha servido para que estos mejoren sus notas finales. También habría que decir (aunque esos datos no se exponen) que aumentó el número de aprobados en junio.

Los alumnos consideran que las principales competencias que ayuda a desarrollar la utilización del portafolio son, por este orden: capacidad de aplicar los conocimientos a la práctica, trabajo en equipos interculturales, gestión del tiempo, capacidad reflexiva, relaciones interpersonales con compañeros y profesores.

Los alumnos en sus encuestas piden que se realicen más veces este tipo de experiencias y que se apliquen a otras asignaturas. Los profesores, han notado que sirve para que los alumnos lleven al día la asignatura, ya que al poner fechas de entrega para cada una de las partes, casi todos los alumnos entregaron los trabajos en dicha fecha. Lo que supuso un gran esfuerzo de corrección para los profesores, y se consiguió uno de los objetivos, que era que los alumnos llevasen al día la asignatura. 
En trabajo adicional para los profesores supuso del orden de 6 horas semanales de corrección, ya que las tutorías se cuentan dentro de las horas establecidas para el profesor.

Los profesores de la asignatura estamos muy satisfechos con los resultados obtenidos y, para los próximos cursos se convertirá el portafolio grupal en un trabajo obligatorio. Aunque somos conscientes de que su utilización conlleva inconvenientes a los que hay que hacer frente, al exigir un elevado nivel de responsabilidad por parte del alumno en la realización de las actividades, ya que tendrán que asistir a tutorías grupales, en las que compartirá con el profesor el desarrollo de su aprendizaje; además, puede llegar a aportar al alumno cierta inseguridad a lo largo del proceso, por el hecho de no estar haciéndolo bien y la carga adicional de trabajo que supone.

Las principales limitaciones encontradas han sido: la falta de experiencia del profesorado en la utilización del portafolio electrónico y, por ello, la excesiva dedicación por parte del profesorado de la asignatura, el trabajo adicional de corrección, así como la necesidad de utilizar herramientas más potentes como Moodle.

Una de las posibles cosas a analizar es el posible impacto al pasar la asignatura de últimos cursos a primeros cursos, pero esto por el momento no es posible ya que necesitaremos más años de impartición de la nueva asignatura.

En definitiva, y aún siendo conscientes de que los resultados de esta experiencia no pueden ser aún concluyentes ni generalizables, consideramos que la utilización del portafolios electrónicos puede ser una técnica válida en la búsqueda de metodologías y sistemas de evaluación acordes con una enseñanza universitaria de calidad.

\section{BIBLIOGRAFÍA}

ARMENGOL, J., HeRnández, J. y MORA, J. [2009]: Experiencias sobre el uso del portafolio del estudiante en la UPC. Revista de Educación a Distancia (RED). Número especial dedicado a Portafolios electrónicos y educación superior. Monográfico VIII, abril-sin mes, 2009, pp. 1-17. http://www.um.es/ead/red/M8.

BARBerÀ, E.; BAUtistA, G.; ESPASA, A. y GUASCH, T. [2006]: Portfolio electrónico: desarrollo de competencias profesionales en la red, Revista de Universidad y Sociedad del Conocimiento (RUSC), vol. III, núm. 2: 55-66.

CABERO, LÓPEZ Y LLORENTE [2012]: E-Portafolio universitario como instrumento didáctico 2.0 para la reflexión, evaluación e investigación de la práctica educativa en el espacio europeo de educación superior. Revista Fundamentos e Investigación VEsC-Año 3- Número 4, año 2012, pp 27-47.

CANO, E. [2005]: El portafolios del profesorado universitario. Un instrumento para la evaluación y para el desarrollo profesional, Editorial Octaedro, S.L. Barcelona.

Clares LóPEZ, J. [2005]: El uso de las TIC en Educación Superior. Actas del XII Congreso Nacional de Modelos de Investigación Educativa, Universidad de La Laguna, del 21 al 23 de septiembre, pp. 243-250.

Colás Bravo, P.S.; Jiménez, R. y VILLACIerVos MOReno, P. [2005]: Portafolios y desarrollo de competencias profesionales en el marco del EEES, Revista de Ciencias de la Educación, núm. 204: 520-538.

COLOMINA, R. Y ROCHERA, M.J. [2002]: Evaluar para ajustar la ayuda educativa. Cuadernos de Pedagogía, vol. 318, noviembre: pp. 56-62.

FLÓREZ R. y ALbELDA, E. [2012]: Una experiencia de trabajo en equipo con soporte documental para la formación de competencias en Contabilidad Financiera. Revista Educade. N³ -AÑO 2012. pp 67-94.

Gallego, D.; CACHeiro, M. L.; MARTín, A.M. y Wilmer, A. [2009]: El portfolio como estrategia de enseñanza y aprendizaje. Revista Electrónica de Tecnología Educativa 
(Edutec), $\quad$ n³0/Noviembre 2009, pp. 1-12. http://edutec.rediris.es/Revelec2/revelec30/articulos_n30_pdf/Edutece30_Gallego_Cacheir_Martin_Angel.pdf.

Celestino GutiérRez, A., Echegaray LegarReta, O. y Guenaga Garal, G. [2003]: Integración de las TIC en la educación superior, Pixel-Bit: Revista de medios y educación, núm. 21:21-28.

García SANZ, M.P. y MORILlas Pedreño, L.R. [2011]: La planificación de evaluación de competencias en Educación Superior, REIFOP, vol. XIV, núm. 1: 113-124.

GARCÍA, B. Y BAENA, R. [2009]: El uso del portafolios en la docencia universitaria: experiencia de renovación metodológica en la asignatura de Geomorfología Fluvial. Revista Iberoamericana de Educación, vol. XLIX, núm. 3: pp. 1-11.

GONZÁLEZ, J. Y WAGENAAR, R. (EDS) (2003): Tuning Educational Structures in Europe. Informe Final. Fase Uno. Bilbao: Universidad de Deusto y Universidad de Groningen.

GuAsCh, T.; GuÀrdiA, L. y BARBERÀ, E. [2009]: Prácticas del portafolio electrónico en el ámbito universitario del Estado Español. Revista de Docencia Universitaria, Monográfico VIII. 30 de Abril de 2009. Número especial dedicado a Portafolios electrónicos y educación superior. http://www.um.es/ead/Red/M8

KIMBALL, M. A. [2002]: The web portfolio guide: Creating electronic portfolios for the web. EUA: Editorial: Longman.

KLENOWSKI, V. [2005]: Desarrollo del portafolios para el aprendizaje y la evaluación. Procesos y principios. Editorial NARCEA, S.A. de ediciones. Madrid. pp. 7-8.

KÖSTER, J. Y RITZEN, M. [2003]: The Amsterdam Faculty of Education's Digital Portfolio- En T. W. Banta (dir.), Portfolio Assessment. Uses, Cases, Scoring, and Impact, vol. 12, n०5, (pp. 24-31), San Francisco: Jossey-Bass.

LÉVY-LEBOYER, C. [1997]: Gestión de las competencias: Cómo analizarlas, cómo evaluarlas, cómo desarrollarlas. Barcelona: Gestión 2.000.

Marín, M., Polo, F. y MATEOS, A. [2011]: "Las metodologías activas en la docencia universitaria de contabilidad". En Las metodologías activas de aprendizaje en la Facultad de Administración y Dirección de Empresas (pp. 15-28.) Valencia: Universitat Politècnica de València.

Martínez Gómez, M. y Marí Benlloch, M. [2011]: "Adaptación de introducción a la estadística al Espacio Europeo de Educación Superior mediante la aplicación de nuevas metodologías docentes". En Las metodologías activas de aprendizaje en la Facultad de Administración de Empresas, Valencia: Universitat Politécnica de València, pp. 95-121.

MATEO ANDRÉS, J. [2007]: Interpretando la realidad, construyendo nuevas formas de conocimiento: el desarrollo competencial y su evaluación. Revista de Investigación Educativa (RIE), vol. XXV, núm. 2: pp. 513-531.

MATEO ANDRÉS, J. y MARTínez OLMO, F. [2005]: L'avaluació alternativa dels aprenentatges. Barcelona: Institut de Ciències de l'Educació de la Universitat de Barcelona. En: http://161.116.7.34/qdu/qdu3.pdf.

MONClús SALAMERO, A.M. [2010]: Una experiencia docente de aplicación del portafolio del estudiante en Contabilidad, Revista Educade, vol. I, núm. 1: pp. 21-42.

Paredes, J. y Estebanell, M. [2005]: Actitudes y necesidades de formación de los profesores antes las TIC y la introducción del crédito europeo: un nuevo desafío para la educación superior, Revista de Educación, núm. 337, mayoagosto: pp. 125-148.

PRendes EsPinosa, M. P. y SÁNChez Vera, M.M. [2008]: Portafolio electrónico: posibilidades para los docentes. Pixel-Bit. Revista de Medios y Educación, núm. 32, marzo: 21- 34.

Zabala Videla, A. y ARnau Belmonte, L. [2007]: 11 ideas clave. Cómo aprender y enseñar competencias, Colección Ideas Clave. Serie Didáctica/Diseño y desarrollo curricular. Editorial GRAÓ, de IRIF, S.L. Barcelona. 